\title{
Strong labelings of linear forests
}

\author{
Martin Bača \\ ${ }^{*}$ Department of Applied Mathematics \\ Technical University in Košice \\ Slovakia \\ Yuqing Lin \\ School of Electrical Engineering and Computer Science \\ The University of Newcastle \\ Callaghan NSW 2308 Australia \\ F. A. Muntaner-Batle \\ ${ }^{\dagger}$ Facultat de Ciències Polítiques i Jurídiques \\ Universitat Internacional de Catalunya, c/ Immaculada 22 \\ 08017 Barcelona, Spain. \\ M. Rius-Font \\ ‡Departament de Matemàtica Aplicada i Telemàtica \\ Universitat Politècnica de Catalunya, Jordi Girona Salgado 1 \\ 08034 Barcelona, Spain. \\ Mathematics Subject Classifications: 05C78,05C05
}

\begin{abstract}
A $(p, q)$-graph $G$ is called super edge-magic if there exists a bijective function $f: V(G) \cup E(G) \rightarrow\{1,2, \ldots, p+q\}$ such that $f(u)+f(v)+f(u v)$ is a constant for each $u v \in E(G)$ and $f(V(G))=$ $\{1,2, \ldots, p\}$.

In this paper, we introduce the concept of strong super edgemagic labeling as a particular class of super edge-magic labelings

\footnotetext{
*Supported by Slovak VEGA Grant 1/4005/07.

${ }^{\dagger}$ Supported in part by Abdus Salam School of Mathematics, Lahore, Pakistan, where part of the research was conducted.

${ }^{\ddagger}$ Supported by the Spanish Research Council under grant BFM2002-00412.
} 
and we use such labelings in order to show that the number of super edge-magic labelings of an odd union of path-like trees, all of them of the same order $m$, grows at least exponentially with $m$.

\section{Introduction.}

Graphs considered in this paper are not necessarily simple, that is, they may contain loops. Also, for most of the graph theory terminology and notation utilized here, the authors refer the reader to Chartrand and Lesniak [12]; however, to make the paper reasonably self contained, we mention that for a graph $G$ we denote the vertex set and edge set of $G$ by $V(G)$ and $E(G)$, respectively.

For a graph or a digraph $D$, we will denote its adjacency matrix by $A(D)$, and for a digraph $D$ we denote by $u n d(D)$ the underlying graph of $D$.

The seminal paper on edge-magic labelings was published in 1970 by Kotzig and Rosa [15], who called these labelings "magic valuations". These were later rediscovered by Ringel and Lladó [19] who coined one of the now popular terms for them: edge-magic labelings. More recently, they have also been refereed to as edge-magic total labelings by Wallis [23]. For a $(p, q)$-graph $G$, a bijective function $f: V(G) \cup E(G) \rightarrow\{1,2, \ldots, p+q\}$ is an edge-magic labeling of $G$ if for each $u v \in E(G), f(u)+f(v)+f(u v)$ is a constant $k$ called the valence of $f$. If such a labeling exists, then $G$ is said to be an edge-magic graph. In [10] Enomoto, Lladó, Nakamigawa and Ringel, defined an edge-magic labeling $f$ of a graph $G$, to be a super edge-magic labeling of $G$ if $f$ has the additional property that $f(V(G))=\{1,2, \ldots, p\}$. Thus, a super edge-magic graph is a graph that admits a super edge-magic labeling. Super edge-magic graphs have been called strong edge-magic total graphs by Wallis [23].

In this paper, we will also consider a path $P_{n}$ to be a particular case of a linear forest and for a linear forest $G$ we introduce the concept of strong super edge-magic labeling as follows: Let $G$ be a $(p, q)$-linear forest, and assume that $f: V(G) \cup E(G) \rightarrow\{1,2, \ldots, p+q\}$ is a super edge-magic labeling of $G$ with the extra property that if $u v \in E(G), u^{\prime} v^{\prime} \notin E(G)$ and $d_{G}\left(u, u^{\prime}\right)=d_{G}\left(v, v^{\prime}\right)<\infty$, then we have that $f(u)+f(v)=f\left(u^{\prime}\right)+$ $f\left(v^{\prime}\right)$. From now on, we will call this property strong. Then, we call $f$ a strong super edge-magic labeling of $G$, and we call $G$ a strong super edgemagic linear forest. For instance, for the path $P_{n}$ as shown in Figure 1 the following labeling $f$ described by Kotzig and Rosa in [15] is in fact a strong 
super edge-magic labeling of the path $P_{n}$.

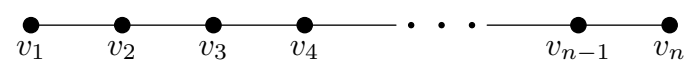

Figure 1: The path $P_{n}$.

$$
\begin{aligned}
f\left(v_{i}\right) & = \begin{cases}\frac{i+1}{2} & \text { if } i \text { is odd } \\
\left\lceil\frac{n}{2}\right\rceil+\frac{i}{2} & \text { if } i \text { is even }\end{cases} \\
f\left(v_{i} v_{i+1}\right) & =2 n-i ; i \in\{1,2, \ldots, n-1\} .
\end{aligned}
$$

At this point, we define the complementary labeling $\bar{f}$ of a strong superedge magic labeling $f$ of a $(p, q)$-linear forest $G$ as follows.

Let $G$ be a $(p, q)$-graph, and let $f: V(G) \cup E(G) \rightarrow\{1,2, \ldots, p+q\}$ be a strong super edge-magic labeling of $G$. The complementary labeling of $f$, denoted by $\bar{f}$, is the labeling defined by the rule

$$
\begin{cases}\bar{f}(v)=p+1-f(v) & \forall v \in V(G) \\ \bar{f}(v w)=2 p+q+1-f(u v) & \forall v w \in E(G) .\end{cases}
$$

One can see that the following lemma is true.

Lemma 1.1 Let $G$ be a $(p, q)$-linear forest and let $f: V(G) \cup E(G) \rightarrow$ $\{1,2, \ldots, p+q\}$ be a strong super edge-magic labeling of $G$. Then the complementary labeling $\bar{f}$ is also a strong super edge-magic labeling of $G$.

Next, consider the following result by Figueroa-Centeno et al. [13].

Lemma 1.2 $A(p, q)$-graph $G$ is super edge-magic if and only if there exists a bijective function $f: V(G) \rightarrow\{1,2, \ldots, p\}$, such that the set

$$
S=\{f(u)+f(v): u v \in E(G)\}
$$

consists of $q$ consecutive integers. In such a case, $f$ can be extended to a super edge-magic labeling of $G$ with valence $k=p+q+s$, where $s=$ $\min (S)$ and

$$
S=\{k-(p+1), k-(p+2), \ldots, k-(p+q)\} .
$$


A graceful labeling of a $(p, q)$-graph $G$ is an injection $g: V(G) \rightarrow\{0,1,2, \ldots$, $q\}$ such that, when each edge $u v$ is assigned the label $|g(u)-g(v)|$, the resulting edge labels (or weights) are distinct. A graph that admits a graceful labeling is said to be graceful. When the graceful labeling $g$ has the property that there exists an integer $\lambda$ such that for each edge $u v$ either $g(u) \leq \lambda<g(v)$ or $g(v) \leq \lambda<g(u), g$ is called an $\alpha$-labeling. The number $\lambda$ is called the boundary value of $g$. A graph with an $\alpha$-labeling is necessarily bipartite and the boundary value must be the smallest of the two vertex labels that yield the edge label 1. A graph that admits an $\alpha$-labeling is called an $\alpha$-graph. Graceful labelings and $\alpha$-labelings are probably the most popular kind among the several classes of the graph labelings. They were introduced by Rosa in [20]. The Ringel-Kotzig conjecture that all trees are graceful is a very popular open problem. Some methods for constructing the graceful labelings and $\alpha$-labelings for certain families of trees can be found in $[3,9,21,22]$.

Let $0 \leq d<n-1$ and let $P_{n}$ be a path with $V\left(P_{n}\right)=\left\{v_{i}: 1 \leq i \leq n\right\}$ and $E\left(P_{n}\right)=\left\{v_{i} v_{i+1}: 1 \leq i \leq n-1\right\}$. Let $f$ be an $\alpha$-labeling of $P_{n}$. Then $f$ will be called an $\alpha_{d}$-labeling of $P_{n}$ if $\min \left\{f\left(v_{1}\right), f\left(v_{n}\right)\right\}=d$. It is known (see [16] or [7]) that if graph $G$ of order $n$ and size $n-1$ admits an $\alpha$-labeling, then $G$ also admits a super edge-magic labeling.

Next lemma gives a relationship between $\alpha_{d}$-labeling of $P_{n}$ for $n$ odd and $d=0,1,2$, and super edge-magic labeling of cycles $C_{n}$.

Lemma 1.3 Let $P_{n}$ be a path on $n$ vertices, $n \geq 3$ odd. If $P_{n}$ admits an $\alpha_{d}$-labeling for $d=0,1,2$, then the cycle $C_{n}$ admits a super edge-magic labeling.

Proof Abrham and Kotzig [1] proved that if $f$ is an $\alpha$-labeling of the path $P_{n}, n=2 t+1$, and $\min \left\{f\left(v_{1}\right), f\left(v_{n}\right)\right\} \leq t-1$, then $f\left(v_{1}\right)+f\left(v_{n}\right)=t=\lambda$. In this case the vertices with the values $>\lambda$ and the vertices with the values $\leq \lambda$ necessarily alternate.

Let $f$ be an $\alpha_{d}$-labeling of $P_{2 t+1}$, for $d=0,1,2$, satisfying $f\left(v_{1}\right)<f\left(v_{n}\right)$ and $f\left(v_{1}\right) \leq t-1$. Consider the following labeling of the vertices of $P_{2 t+1}$ :

$g\left(v_{i}\right)=\left\{\begin{array}{lll}f\left(v_{i}\right)+1 & \text { if } \quad i \quad \text { is even } \\ t+1-f\left(v_{i}\right) & \text { if } \quad i \quad \text { is odd. }\end{array}\right.$

Since $f\left(v_{1}\right) \leq t-1=\lambda-1$, then the labels assigned by $g$ to the vertices $v_{i}$, $i$ odd, are $1,2, \ldots, \lambda, \lambda+1$, and those assigned to the vertices $v_{i}, i$ even, are $\lambda+2, \lambda+3, \ldots, n$. Thus, $g$ is an injection from $V\left(P_{n}\right)$ onto $\{1,2, \ldots, n\}$. Furthermore $\left\{\left|f\left(v_{i}\right)-f\left(v_{i+1}\right)\right|: i=1,2, \ldots, n-1\right\}=\{1,2, \ldots, n-1\}$ since 
$f$ is an $\alpha$-labeling and $\left\{g\left(v_{i}\right)+g\left(v_{i+1}\right)=t+2+\left|f\left(v_{i}\right)-f\left(v_{i+1}\right)\right|: i=\right.$ $1,2, \ldots, n-1\}=\{t+3, t+4, \ldots, 3 t+2\}$.

If $d=0$ then $f\left(v_{1}\right)=0, f\left(v_{n}\right)=t$ and $g\left(v_{1}\right)=t+1, g\left(v_{n}\right)=1$.

If $d=1$ then $f\left(v_{1}\right)=1, f\left(v_{n}\right)=t-1$ and $g\left(v_{1}\right)=t, g\left(v_{n}\right)=2$.

If $d=2$ then $f\left(v_{1}\right)=2, f\left(v_{n}\right)=t-2$ and $g\left(v_{1}\right)=t-1, g\left(v_{n}\right)=3$.

For each previous case $g\left(v_{1}\right)+g\left(v_{n}\right)=t+2$ and according to Lemma 1.2 the vertex labeling $g$ can be extend to a super edge-magic labeling of cycle $C_{n}$ with valence $5 t+4$.

From now on, the symbol $N_{d}(n)$ will be used to denote the number of $\alpha_{d^{-}}$ labeling of $P_{n}$. The next lemma gives an exponential lower bound for the number of super edge-magic labelings of the cycle $C_{n}, n$ odd.

Lemma 1.4 Let $C_{n}$ be a cycle on $n$ vertices, $n \geq 11$ odd. The number of super edge-magic labelings of the cycle $C_{n}$ is at least $\frac{5}{4} 2^{\left\lfloor\frac{n}{3}\right\rfloor}+1$.

Proof Abrham and Kotzig [1] proved that $N_{0}(n)=1$ for every $n \geq$ $2, N_{1}(n) \geq \frac{1}{4} 2^{\left\lfloor\frac{n}{3}\right\rfloor}$ for every $n \geq 6$, and $N_{2}(n) \geq 2^{\left\lfloor\frac{n}{3}\right\rfloor}$ for every $n \geq 10$ . With respect to Lemma 1.3 and Abrham and Kotzig result, we have that for every $n \geq 11$ odd, the number of super edge-magic labelings of the cycle $C_{n}$ is at least $N_{0}(n)+N_{1}(n)+N_{2}(n) \geq \frac{5}{4} 2^{\left\lfloor\frac{n}{3}\right\rfloor}+1$.

At this point, we will state a version of Lemma 1.2 for strong super edgemagic labelings, which follows immediately from Lemma 1.2 and the definition of strong super edge-magic labeling.

Lemma 1.5 $A(p, q)$-linear forest $G$ is strong super edge-magic if and only if there exists a bijective function $f: V(G) \rightarrow\{1,2, \ldots, p\}$, such that:

1. the set $S=\{f(u)+f(v): u v \in E(G)\}$ consists of $q$ consecutive integers,

2. if $u v \in E(G)$ and $d_{G}\left(u, u^{\prime}\right)=d_{G}\left(v, v^{\prime}\right)<\infty$ for two vertices $u^{\prime}, v^{\prime} \in$ $V(G)$, and $u^{\prime} v^{\prime} \notin E(G)$, then $f(u)+f(v)=f\left(u^{\prime}\right)+f\left(v^{\prime}\right)$.

In such a case, $f$ can be extended to a strong super edge-magic labeling of $G$ with valence $k=p+q+s$, where $s=\min (S)$ and

$S=\{f(u)+f(v): u v \in E(G)\}=\{k-(p+1), k-(p+2), \ldots, k-(p+q)\}$. 
Thus, due to Lemma 1.5, it is sufficient to exhibit the vertex labels of a strong super edge-magic labeling.

In his Ph.D. thesis, Barrientos [8] introduced the concept of a path-like tree as follows:

We embed the path $P_{n}$ as a subgraph of the 2-dimensional grid, this is to say the cartesian product $P_{r} \times P_{t}$ of a path on $r$ vertices with a path on $t$ vertices. Given such an embedding, we consider the ordered set of subpaths $L_{1}, L_{2}, \ldots, L_{h}$ which are maximal straight segments in the embedding, where the end of $L_{i}$ is the beginning of $L_{i+1}$ for any $i=1,2, \ldots, h-1$. Suppose that $L_{i} \cong P_{2}$ for some $i, 1<i<h, V\left(L_{i}\right)=\left\{u_{0}, v_{0}\right\}$, thus $u_{0} \in V\left(L_{i-1}\right) \cap V\left(L_{i}\right)$ and $v_{0} \in V\left(L_{i}\right) \cap V\left(L_{i+1}\right)$. Let $u \in V\left(L_{i-1}\right)-\left\{u_{o}\right\}$ and $v \in V\left(L_{i+1}\right)-\left\{v_{o}\right\}$ such that their distance on the grid is 1 . The replacement of the edge $u_{0} v_{0}$ by the new edge $u v$ is called an elementary transformation of the path $P_{n}$. We say that a tree $T$ of order $n$ is a path-like tree, when it can be obtained after a sequence of elementary transformations on an embedding of $P_{n}$ in the 2-dimensional grid.

The concept of path-like tree is very similar to the concept of $T_{p}$-tree. Although in [17] it was shown that the two concepts are different. For further information on $T_{p}$-trees, the interested reader may consult [2], [11] and [17].

The labeling properties of path-like trees have been studied by many authors lately, for instance Barrientos [8], Bača et al. [4], [5], [6] and Ngurah et al. [18].

In a recent work Bača et al. [5] studied the super edge-magic properties of an odd number of copies of path-like trees and they proved the following result.

Theorem 1.6 Let $T_{j}, 1 \leq j \leq m$, be a path-like tree of order $n$. If $m$ is odd, $m \geq 3$ and $n \geq 4$, then a forest $F \cong \bigcup_{j=1}^{m} T_{j}$ admits a super edge-magic labeling.

It is clear that if $m P_{n}$ is strong super edge-magic for $m$ odd, then the forest $F \cong \bigcup_{j=1}^{m} T_{j}$, where each $T_{j}$ is a path-like tree of order $n$, is super edgemagic. The authors in [5] provided a strong super edge-magic labeling of $m P_{n}$ for $m$ odd and it was the technique used in order to prove Theorem 1.6. The main goal of this paper is to show that the number of non-isomorphic strong super edge-magic labelings of the graph $m P_{n}$, for $m$ odd and any $n$, grows very fast with $m$. This allows us to generate an exponential number of non-isomorphic super edge-magic labelings of the forest $F \cong \bigcup_{j=1}^{m} T_{j}$, where each $T_{j}$ is a path-like tree of order $n$ and $m$ is an odd integer. We do 
this, using a technique introduced in [14] that involves products of digraphs. In the next lines we will describe this digraph operation.

Let $D$ be a digraph and let $\Gamma=\left\{F_{1}, F_{2}, \ldots, F_{s}\right\}$ be a family of digraphs that meet the following conditions:

1) $V\left(F_{i}\right)=V$ for every $i \in\{1,2, \ldots, s\}$.

2) $\operatorname{out}(v)=i n(v)=1$ for every $v \in V\left(F_{i}\right)$.

3) $F_{i}$ may contain loops. In other words, each $F_{i}$ of the same order is either a cycle or union of cycles (possibly loops) such that each component has been oriented cyclically.

4) Each vertex of $F_{i}$ takes the name of a super edge-magic labeling of $F_{i}$.

Consider any function $h: E(D) \rightarrow \Gamma$. Then the product $D \bigotimes_{h} \Gamma$ is a digraph with vertex set $V\left(D \otimes_{h} \Gamma\right)=V(D) \times V$ and

$((a, b),(c, d)) \in E\left(D \bigotimes_{h} \Gamma\right) \Longleftrightarrow[(a, c) \in E(D) \wedge(b, d) \in E(h(a, c))]$.

Notice that the adjacency matrix of $D \bigotimes_{h} \Gamma$, denoted by $A\left(D \bigotimes_{h} \Gamma\right)$, is obtained by multiplying every 0 entry of $A(D)$, where $A(D)$ denotes the adjacency matrix of $D$, by the $|V| \times|V|$ null square matrix, and every 1 entry of $A(D)$ by $A(h(a, c))$, where $A(h(a, c))$ denotes the adjacency matrix of $h(a, c)$. Notice that when the function $h$ is constant, we have the classical Kronecker matrix product.

In [14] Figueroa-Centeno et al. call a digraph $D$ super edge-magic if und $(D)$ is super edge-magic, and they proved the following two results:

Theorem 1.7 Let $D$ be a super edge-magic digraph for which each vertex takes the name of its label. Let $\Gamma=\left\{F_{1}, F_{2}, \ldots, F_{s}\right\}$ be a family of all super edge-magic 1 -regular labeled digraphs (each $F_{i}, 1 \leq i \leq s$, is either a cycle or union of cycles such that each component has been oriented cyclically) of the same odd order each, where each vertex of $F_{i}, 1 \leq i \leq s$, takes the name of its label. Consider any function $h: E(D) \longrightarrow \Gamma$. Then the digraph $D \otimes_{h} \Gamma$ is super edge-magic.

Theorem 1.8 Let $\vec{T}$ be any oriented tree. Let $\Gamma=\left\{F_{1}, F_{2}, \ldots, F_{s}\right\}$ be a family of 1-regular digraphs of order $m$ each. Consider any function $h: E(\vec{T}) \longrightarrow \Gamma$. Then und $\left(\vec{T} \bigotimes_{h} \Gamma\right)=m T$.

As we already said before, in the paper we use this operation, in order to obtaining an exponential lower bound for the number of strong super 
edge-magic labelings of the union of paths, starting from the strong super edge-magic labeling of the path provided in [15].

\section{Generating strong super edge-magic labelings for $m P_{n}, m$ odd.}

In this section, we will describe an algorithm, that will allow us to create strong super edge-magic labelings for the graph $m P_{n}, m=2 K+1, K, n \in$ $N$. Then, we will illustrate the algorithm with an specific example.

Input:

1. Oriented path $\overrightarrow{P_{n}}$ with:

- Vertex set $V\left(\overrightarrow{P_{n}}\right)=\left\{v_{i}\right\}_{i=1}^{n}$ and $E\left(\vec{P}_{n}\right)=\left\{\left(v_{i} v_{i+1}\right)\right\}_{i=1}^{n-1}$

- Consider a function $f: V\left(\vec{P}_{n}\right) \longrightarrow\{1,2, \ldots, n\}$ defined by the rule:

$$
f\left(v_{i}\right)= \begin{cases}\frac{i+1}{2}, & \text { if } i \text { is odd } \\ \left\lceil\frac{n}{2}\right\rceil+\frac{i}{2}, & \text { if } i \text { is even . }\end{cases}
$$

Observation: The labeling $f$, which is a strong super-edge magic labeling of $\vec{P}_{n}$, could be substituted by any strong super edge-magic labeling of the oriented path $\vec{P}_{n}$.

2. The set $\Gamma_{m}=\left\{F_{1}, F_{1}^{\prime}, F_{2}, F_{2}^{\prime}, \ldots, F_{\frac{s}{2}}, F_{\frac{s}{2}}^{\prime}\right\}$ is the family of all 1 -regular digraphs where each digraph of order $m=2 K+1$ is labeled in a super edge-magic way, and each vertex takes the name of its label. Each couple $\left(F_{j}, F_{j}^{\prime}\right)$ comes from the same underlying 2-regular graph, but it has been oriented in opposite way. That is to say, if a component is oriented clockwise in $F_{j}$, then the corresponding component is oriented counter clockwise in $F_{j}^{\prime}$, and viceversa.

3. A function $h: E\left(\vec{P}_{n}\right) \longrightarrow \Gamma_{m}$ with

$$
h\left(v_{i-1} v_{i}\right)= \begin{cases}F_{j}, & \text { if } i \text { is even } \\ F_{j}^{\prime}, & \text { if } i \text { is odd }\end{cases}
$$

for any fixed $j \in\left\{1,2, \ldots, \frac{s}{2}\right\}$.

Observation: $\left\{\begin{array}{ll}f\left(v_{i-1}\right)=x \\ f\left(v_{i}\right)=x^{\prime}\end{array} \Longrightarrow h\left(v_{i-1} v_{i}\right)= \begin{cases}F_{j}, & \text { if } x+x^{\prime}+\left\lceil\frac{n}{2}\right\rceil \text { is even. } \\ F_{j}^{\prime}, & \text { if } x+x^{\prime}+\left\lceil\frac{n}{2}\right\rceil \text { is odd }\end{cases}\right.$ 
Algorithm:

1. Rename each vertex of $\vec{P}_{n}$ with the name of its label, creating a new graph $\vec{P}_{n}^{l}$.

2. Compute $\vec{P}_{n}^{l} \bigotimes_{h} \Gamma_{m}=\vec{Q}$.

3. Take $\operatorname{und}(\vec{Q})=Q$.

4. Let $\left(x_{i}, y_{i}\right) \in V(Q)$. Relabel the vertex $\left(x_{i}, y_{i}\right)$ with $z_{i}$ where $z_{i}$ is computed using the formula

$$
z_{i}=m\left(x_{i}-1\right)+y_{i}
$$

creating the new graph $Q^{l}$.

Output:

$Q^{l}=(2 K+1) P_{n}$ labeled in a strong super edge-magic way.

Proof By theorems 1.7 and 1.8, it is known that $Q^{l}$ is super edge-magic and that $Q^{l} \cong(2 K+1) P_{n}$. It only remains to be shown that the obtained labeling preserves the "strong property".

Let $(x, y),\left(x^{\prime}, y^{\prime}\right)$ and $\left(x^{\prime \prime}, y^{\prime \prime}\right)$ be the three vertices of $Q$ such that

$$
\left.\begin{array}{l}
\left\{(x, y),\left(x^{\prime}, y^{\prime}\right)\right\} \\
\left\{\left(x^{\prime}, y^{\prime}\right),\left(x^{\prime \prime}, y^{\prime \prime}\right)\right\}
\end{array}\right\} \in E(Q) .
$$

If $x+x^{\prime}$ is odd, then $x^{\prime}+x^{\prime \prime}$ is even and viceversa. Since $F_{j}$ and $F_{j}^{\prime}$ have opposite orientations, we obtain that $y=y^{\prime \prime}$.

Let $\left(x_{i}, y_{i}\right), 1 \leq i \leq n$ be the labels of the $n$ "consecutive" vertices of a component of $Q$. By the previous observation we have that $y_{i}=y_{j}$ if $|i-j|$ is even. Hence $y_{i}+y_{i+1}=y_{i-r}+y_{i+r+1}$ for every $i \in\{1,2, \ldots, n-2\}$ $(r \leq \min \{i-1, n-i-1,1\})$.

Now, following the notation introduced in the algorithm, we denote by $z_{i}$ the vertex of $Q^{l}$ that corresponds to the vertex $\left(x_{i}, y_{i}\right)$ in $Q$. We want to show that

$$
z_{i}+z_{i+1}=z_{i-r}+z_{i+1+r} .
$$

Notice that:

$$
\left.\begin{array}{ll}
z_{i}+z_{i+1} & =m\left[x_{i}+x_{i+1}-2\right]+\left(y_{i}+y_{i+1}\right) \\
z_{i-r}+z_{i+1+r} & =m\left[x_{i-r}+x_{i+1+r}-2\right]+\left(y_{i-r}+y_{i+1+r}\right)
\end{array}\right\},
$$


- $y_{i}+y_{i+1}=y_{i-r}+y_{i+1+r}$ by previous argument,

- $x_{i}+x_{i+1}=x_{i-r}+x_{i+1+r}$ since these are the same labels that we used in the oriented path $\vec{P}_{n}^{l}$, and the labeling of $\vec{P}_{n}^{l}$ is a strong super edge-magic labeling.

Therefore,

$$
z_{i}+z_{i+1}=z_{i-r}+z_{i+1+r}
$$

In the following example we use the previous algorithm in order to obtain a strong super edge-magic labeling of $5 P_{6}$. Let $\vec{P}_{6}$ be the following digraph, where each vertex of $\vec{P}_{6}$ takes the name of the strong super edge-magic labeling described in the algorithm.

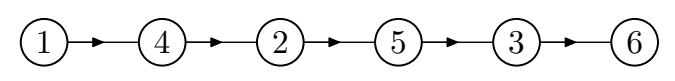

Figure 2: Vertex labeling of the digraph $\vec{P}_{6}$.

Let $\Gamma_{5}=\left\{F_{1}, F_{1}^{\prime}, F_{2}, F_{2}^{\prime}, F_{3}, F_{3}^{\prime}\right\}$ be the family of all super edge-magic 1 - regular digraphs of order 5 such that each component is oriented cyclically and each vertex of each digraph takes the name of a super edge-magic labeling. Figures 3-14 illustrate the digraphs and their corresponding adjacency matrices.

Figure 15 depicts the adjacency matrix of the digraph $\vec{P}_{6}$.

Now, define the function $h: E\left(\vec{P}_{6}\right) \longrightarrow\left\{F_{1}, F_{1}^{\prime}\right\}$ such that for every edge

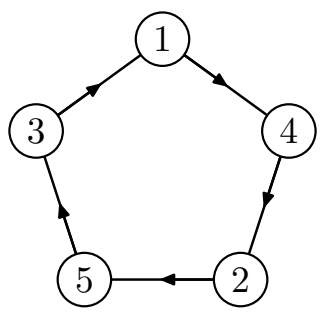

Figure 3: Digraph $F_{1}$.

$$
A\left(F_{1}\right)=\begin{array}{c|ccccc} 
& 1 & 2 & 3 & 4 & 5 \\
\hline 1 & 0 & 0 & 0 & 1 & 0 \\
2 & 0 & 0 & 0 & 0 & 1 \\
3 & 1 & 0 & 0 & 0 & 0 \\
4 & 0 & 1 & 0 & 0 & 0 \\
5 & 0 & 0 & 1 & 0 & 0
\end{array}
$$

Figure 4: Adjacency matrix of $F_{1}$. 


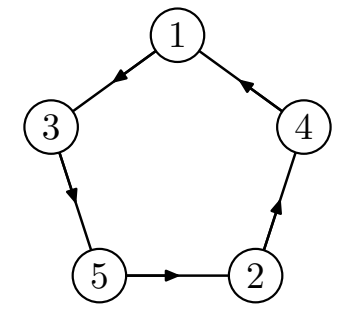

Figure 5: Digraph $F_{1}^{\prime}$.

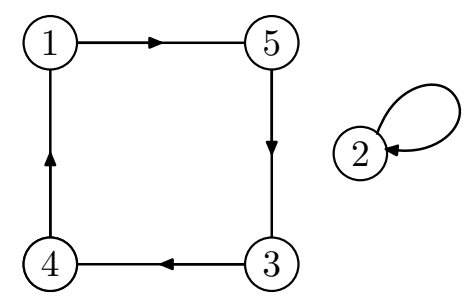

Figure 7: Digraph $F_{2}$.
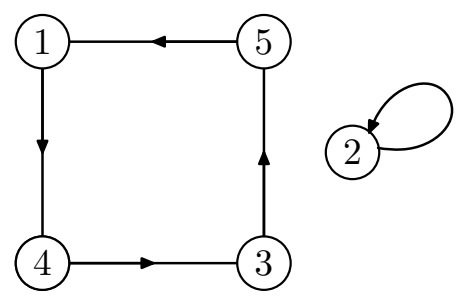

Figure 9: Digraph $F_{2}^{\prime}$.
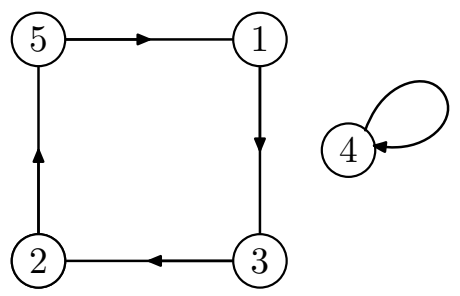

Figure 11: Digraph $F_{3}$.

$$
A\left(F_{1}^{\prime}\right)=\begin{array}{c|ccccc} 
& 1 & 2 & 3 & 4 & 5 \\
\hline 1 & 0 & 0 & 1 & 0 & 0 \\
2 & 0 & 0 & 0 & 1 & 0 \\
3 & 0 & 0 & 0 & 0 & 1 \\
4 & 1 & 0 & 0 & 0 & 0 \\
5 & 0 & 1 & 0 & 0 & 0
\end{array}
$$

Figure 6: Adjacency matrix of $F_{1}^{\prime}$.

$$
A\left(F_{2}\right)=\begin{array}{c|ccccc} 
& 1 & 2 & 3 & 4 & 5 \\
\hline 1 & 0 & 0 & 0 & 0 & 1 \\
2 & 0 & 1 & 0 & 0 & 0 \\
3 & 0 & 0 & 0 & 1 & 0 \\
4 & 1 & 0 & 0 & 0 & 0 \\
5 & 0 & 0 & 1 & 0 & 0
\end{array}
$$

Figure 8: Adjacency matrix of $F_{2}$.

$$
A\left(F_{2}^{\prime}\right)=\begin{array}{c|ccccc} 
& 1 & 2 & 3 & 4 & 5 \\
\hline 1 & 0 & 0 & 0 & 1 & 0 \\
2 & 0 & 1 & 0 & 0 & 0 \\
3 & 0 & 0 & 0 & 0 & 1 \\
4 & 0 & 0 & 1 & 0 & 0 \\
5 & 1 & 0 & 0 & 0 & 0
\end{array}
$$

Figure 10: Adjacency matrix of $F_{2}^{\prime}$.

$$
A\left(F_{3}\right)=\begin{array}{c|ccccc} 
& 1 & 2 & 3 & 4 & 5 \\
\hline 1 & 0 & 0 & 1 & 0 & 0 \\
2 & 0 & 0 & 0 & 0 & 1 \\
3 & 0 & 1 & 0 & 0 & 0 \\
4 & 0 & 0 & 0 & 1 & 0 \\
5 & 1 & 0 & 0 & 0 & 0
\end{array}
$$

Figure 12: Adjacency matrix of $F_{3}$. 


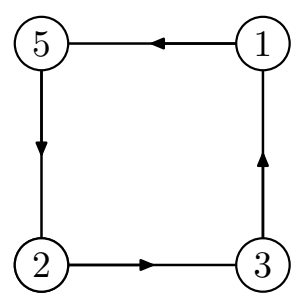

Figure 13: Digraph $F_{3}^{\prime}$.

$$
A\left(F_{3}^{\prime}\right)=\begin{array}{c|ccccc} 
& 1 & 2 & 3 & 4 & 5 \\
\hline 1 & 0 & 0 & 0 & 0 & 1 \\
2 & 0 & 0 & 1 & 0 & 0 \\
3 & 1 & 0 & 0 & 0 & 0 \\
4 & 0 & 0 & 0 & 1 & 0 \\
5 & 0 & 1 & 0 & 0 & 0
\end{array}
$$

Figure 14: Adjacency matrix of $F_{3}^{\prime}$.

$$
A\left(\overrightarrow{P_{6}}\right)=\begin{array}{c|cccccc} 
& 1 & 2 & 3 & 4 & 5 & 6 \\
\hline 1 & 0 & 0 & 0 & 1 & 0 & 0 \\
2 & 0 & 0 & 0 & 0 & 1 & 0 \\
3 & 0 & 0 & 0 & 0 & 0 & 1 \\
4 & 0 & 1 & 0 & 0 & 0 & 0 \\
5 & 0 & 0 & 1 & 0 & 0 & 0 \\
6 & 0 & 0 & 0 & 0 & 0 & 0
\end{array}
$$

Figure 15: Adjacency matrix of $\vec{P}_{6}$.

$x x^{\prime} \in E\left(\vec{P}_{6}\right)$, we put

$$
h\left(x, x^{\prime}\right)= \begin{cases}F_{j}, & \text { if } x+x^{\prime} \equiv 1(\bmod 2) \\ F_{j}^{\prime}, & \text { if } x+x^{\prime} \equiv 0(\bmod 2)\end{cases}
$$

The adjacency matrix of $\vec{P}_{6} \bigotimes_{h}\left\{F_{1}, F_{1}^{\prime}\right\}$ is obtained by multiplying every 0 entry of $A\left(\vec{P}_{6}\right)$ by the $5 \times 5$ null square matrix and every 1 entry of $A\left(\vec{P}_{6}\right)$ by $A\left(F_{1}\right)$ or $A\left(F_{1}^{\prime}\right)$, see Figure 16 .

The underlying graph of $\vec{P}_{6} \bigotimes_{h}\left\{F_{1}, F_{1}^{\prime}\right\}$ is isomorphic to $5 P_{6}$. The adjacency matrix $A\left(\vec{P}_{6} \bigotimes_{h}\left\{F_{1}, F_{1}^{\prime}\right\}\right)$ describes the corresponding vertex labeling of a strong super edge-magic labeling of $5 P_{6}$, see Figure 17.

Let $m$ be an odd positive integer, $m \geq 3$, and denote by $N(m)$ the number of non-isomorphic strong super edge-magic labelings of the graph $m P n$, $n \geq 4$. The next theorem gives an exponential lower bound for $N(m)$.

Theorem 2.1 Let $m \geq 5$ be an odd integer. Then $N(m) \geq \frac{5}{2} 2^{\left\lfloor\frac{m}{3}\right\rfloor}+1$.

Proof In [14] (see Lemma 4.1) it was shown that if $h: E(D) \longrightarrow \Gamma$ and 


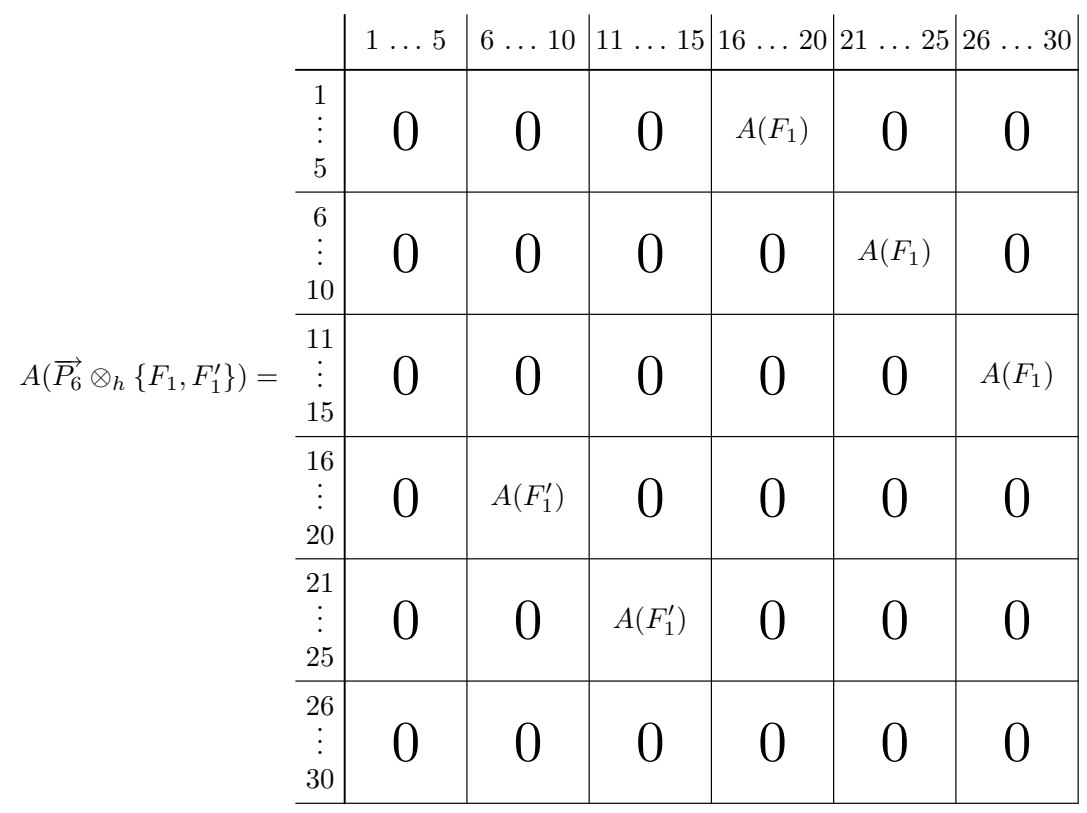

Figure 16: Adjacency matrix of $\vec{P}_{6} \bigotimes_{h}\left\{F_{1}, F_{1}^{\prime}\right\}$.

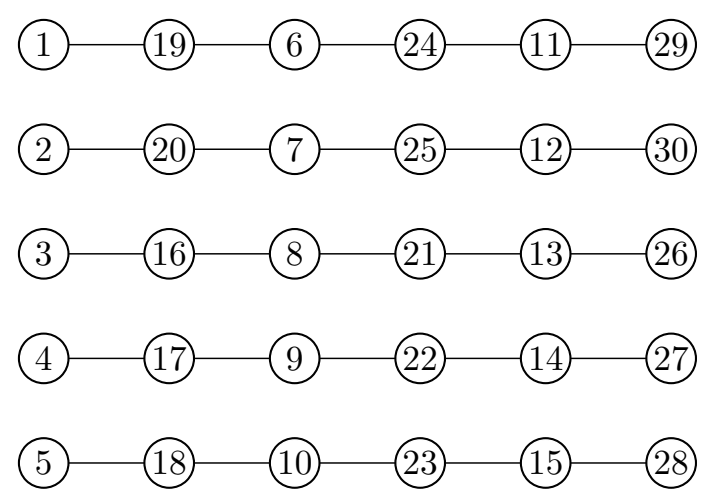

Figure 17: Vertex labeling of a strong super edge-magic labeling of $5 P_{6}$. 
$h^{\prime}: E(D) \longrightarrow \Gamma$ are two different functions, then the labelings obtained applying the product are non-isomorphic.

Consider a strong super edge-magic labeling of $P_{n}, n \geq 4$, and $\Gamma_{m}=$ $\left\{F_{1}, F_{1}^{\prime}, F_{2}, F_{2}^{\prime}, \ldots, F_{\frac{s}{2}}, F_{\frac{s}{2}}^{\prime}\right\}$ as a family of super edge-magic 1-regular digraphs of order $m$. The family $\Gamma_{m}$ consists of $\frac{s}{2}$ couples of the same super edge-magic labeled 1-regular digraphs $\left(F_{j}, F_{j}^{\prime}\right), j=1,2, \ldots, \frac{s}{2}$, but with opposite orientations. With respect to the previous algorithm for generating strong super edge-magic labelings of the union of paths $m P_{n}$ there are $s$ different functions $h_{j}: E(D) \longrightarrow\left\{F_{j}, F_{j}^{\prime}\right\}$, for $j=1,2, \ldots, \frac{s}{2}$ (each couple $\left(F_{j}, F_{j}^{\prime}\right)$ has two possible orientations), and also $s$ non-isomorphic strong super edge-magic labelings of $m P_{n}$.

It remains to investigate how many different couples $\left(F_{j}, F_{j}^{\prime}\right)$ contains the family $\Gamma_{m}$.

Let us distinguish the four following cases, according to the order $m$.

Case 1. $m=5$. We have three couples $\left(F_{1}, F_{1}^{\prime}\right),\left(F_{2}, F_{2}^{\prime}\right)$ and $\left(F_{3}, F_{3}^{\prime}\right)$, see Figures $3,5,7,9,11$ and 13 . With respect to two possible orientations of each couple we can see that in this case the lower bound is tight.

Case 2. $m=7$. There are at least 14 couples. They are described in [14]. Let us rewrite them in Table 1.

\begin{tabular}{|lcc|}
\hline graph & vertex labeling & $\begin{array}{c}\text { number of possible } \\
\text { orientations }\end{array}$ \\
\hline$C_{5} \cup C_{1} \cup C_{1}$ & $1-4-7-2-6 \cup 3 \cup 5$ & 2 \\
$C_{6} \cup C_{1}$ & $1-6-3-2-4-7 \cup 5$ & 2 \\
$C_{6} \cup C_{1}$ & $1-4-6-5-2-7 \cup 3$ & 2 \\
$C_{3} \cup C_{3} \cup C_{1}$ & $1-5-6 \cup 2-3-7 \cup 4$ & 4 \\
$C_{7}$ & $1-5-2-6-3-7-4$ & 2 \\
$C_{7}$ & $1-6-5-3-7-2-4$ & 2 \\
$C_{7}$ & $1-7-3-6-5-2-4$ & 2 \\
$C_{7}$ & $1-4-3-7-2-6-5$ & 2 \\
$C_{7}$ & $1-7-2-3-4-6-5$ & 2 \\
$C_{7}$ & $1-6-4-7-2-3-5$ & 2 \\
$C_{7}$ & $1-6-2-3-7-4-5$ & 2 \\
$C_{7}$ & $1-5-2-3-6-4-7$ & 2 \\
$C_{7}$ & $1-6-5-4-2-3-7$ & 2 \\
\hline
\end{tabular}

Table 1: Super edge-magic 2-regular graphs of order 7 .

Case 3. $m=9$. There are at least 39 couples. Table 2 shows these super edge-magic 2-regular graphs of order 9 , where each component has been oriented cyclically. 


\begin{tabular}{|llc|}
\hline graph & vertex labeling & $\begin{array}{c}\text { number } \\
\text { of possible } \\
\text { orientations }\end{array}$ \\
\hline$C_{3} \cup C_{3} \cup C_{3}$ & $1-5-9 \cup 2-6-7 \cup 3-4-8$ & 8 \\
$C_{3} \cup C_{3} \cup C_{3}$ & $1-6-8 \cup 2-4-9 \cup 3-5-7$ & 8 \\
$C_{5} \cup C_{4}$ & $1-5-2-6-8 \cup 3-7-4-9$ & 4 \\
$C_{5} \cup C_{4}$ & $2-9-5-8-4 \cup 1-6-3-7$ & 4 \\
$C_{5} \cup C_{3} \cup C_{1}$ & $2-9-5-4-8 \cup 1-6-7 \cup 3$ & 4 \\
$C_{5} \cup C_{3} \cup C_{1}$ & $1-7-6-4-8 \cup 2-5-9 \cup 3$ & 4 \\
$C_{5} \cup C_{3} \cup C_{1}$ & $1-5-6-2-8 \cup 3-4-9 \cup 7$ & 4 \\
$C_{5} \cup C_{3} \cup C_{1}$ & $2-6-4-3-9 \cup 1-5-8 \cup 7$ & 4 \\
$C_{6} \cup C_{1} \cup C_{1} \cup C_{1}$ & $1-6-2-9-4-8 \cup 3 \cup 5 \cup 7$ & 2 \\
$C_{7} \cup C_{1} \cup C_{1}$ & $1-7-4-9-5-2-8 \cup 3 \cup 6$ & 2 \\
$C_{7} \cup C_{1} \cup C_{1}$ & $1-5-8-2-9-3-6 \cup 4 \cup 7$ & 2 \\
$C_{7} \cup C_{1} \cup C_{1}$ & $1-6-8-4-9-2-7 \cup 3 \cup 5$ & 2 \\
$C_{7} \cup C_{1} \cup C_{1}$ & $1-8-3-9-4-2-6 \cup 5 \cup 7$ & 2 \\
$C_{7} \cup C_{1} \cup C_{1}$ & $1-9-2-5-7-6-8 \cup 3 \cup 4$ & 2 \\
$C_{7} \cup C_{1} \cup C_{1}$ & $1-8-5-3-4-2-9 \cup 6 \cup 7$ & 2 \\
$C_{8} \cup C_{1}$ & $1-9-4-7-5-2-6-8 \cup 3$ & 2 \\
$C_{8} \cup C_{1}$ & $1-5-2-7-6-8-3-9 \cup 4$ & 2 \\
$C_{8} \cup C_{1}$ & $1-7-2-4-9-3-8-6 \cup 5$ & 2 \\
$C_{8} \cup C_{1}$ & $1-9-5-8-3-4-2-7 \cup 6$ & 2 \\
$C_{8} \cup C_{1}$ & $1-6-3-5-8-4-2-9 \cup 7$ & 2 \\
$C_{9}$ & $1-6-2-7-3-8-4-9-5$ & 2 \\
$C_{9}$ & $1-5-7-2-6-8-3-4-9$ & 2 \\
$C_{9}$ & $1-5-9-2-6-7-3-4-8$ & 2 \\
$C_{9}$ & $1-5-7-3-4-9-2-6-8$ & 2 \\
$C_{9}$ & $1-6-7-3-5-9-2-4-8$ & 2 \\
$C_{9}$ & $1-6-7-2-4-8-3-5-9$ & 2 \\
$C_{9}$ & $1-6-8-3-5-7-2-4-9$ & 2 \\
\hline & & \\
& &
\end{tabular}

Table 2: Super edge-magic 2-regular graphs of order 9.

Case 4. $m \geq 11$. If we consider only super edge-magic cyclically oriented cycles of order $m$, as elements of the family $\Gamma_{m}$, then from Lemma 1.4 it follows that, for $m \geq 11$, there exist at least $\frac{5}{4} 2^{\left\lfloor\frac{m}{3}\right\rfloor}+1$ couples, where each couple comes from the same super edge-magic labeled cycle but with opposite orientations.

Since in the last three cases each couple of oriented cycles has two possible orientations, then there is at least $\frac{5}{2} 2^{\left\lfloor\frac{m}{3}\right\rfloor}+1$ non-isomorphic strong super edge-magic labelings of the graph $m P_{n}$.

According to the previous two cases, for $m=7$ and 9 , we can observe that 


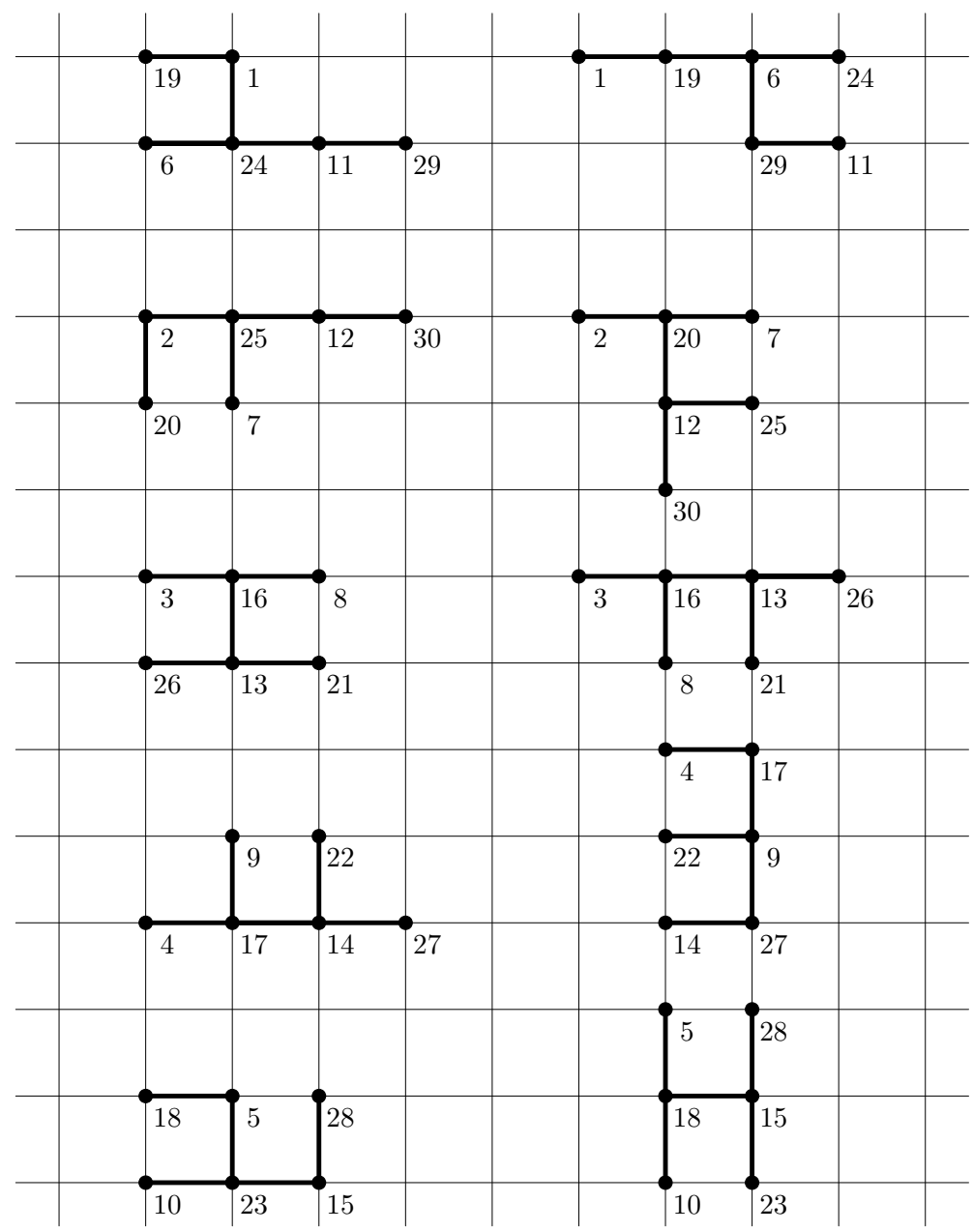

Figure 18: Vertex labeling of super edge-magic labelings of $\bigcup_{j=1}^{5} T_{j}$. 
there exist also super edge-magic 1-regular disconnected graphs of order $m$. It means that really the lower bound of Theorem 2.1 is bigger.

Theorem 2.2 Let $P_{n}$ be strong super edge-magic for $n \geq 4$. If $m$ is odd, $m \geq 5$, then a forest $F \cong \bigcup_{j=1}^{m} T_{j}$, where each $T_{j}$ is a path-like tree of order $n$, admits at least $\frac{5}{2} 2^{\left\lfloor\frac{m}{3}\right\rfloor}+1$ non-isomorphic super edge-magic labelings.

Proof From Theorem 2.1 it follows that if $P_{n}$ dmits a strong super edgemagic labeling then there are at least $\frac{5}{2} 2^{\left\lfloor\frac{m}{3}\right\rfloor}+1$ non-isomorphic strong super edge-magic labelings of the disjoint union of paths $\bigcup_{j=1}^{m} P_{j}$ for $m$ odd.

Consider an embedding of the disjoint union of paths $P_{1}, P_{2}, \ldots, P_{m}$ in the 2 -dimensional grid. Let $P_{j}=T_{j}^{0}, T_{j}^{1}, T_{j}^{2}, \ldots, T_{j}^{s_{j}}=T_{j}$ be the series of trees obtained by successively applying the appropriate elementary transformations of $P_{j}$ to obtain $T_{j}$, for $j=1,2, \ldots, m$, which keep the super edge-magic character of the path $P_{j}$.

There are different series of trees $T_{j}^{0}, T_{j}^{1}, T_{j}^{2}, \ldots, T_{j}^{s_{j}}$ for different $s_{j}$, i.e., the forest $F$ is a disjoint union of different path-like trees $T_{1}, T_{2}, \ldots, T_{m}$, each of order $n$. For each strong super edge-magic labeling of $\bigcup_{j=1}^{m} P_{j}, m$ odd, there exists a super edge-magic labeling of the forest $F \cong \bigcup_{j=1}^{m} T_{j}$.

Thus, the forest $F \cong \bigcup_{j=1}^{m} T_{j}$ admits at least $\frac{5}{2} 2^{\left\lfloor\frac{m}{3}\right\rfloor}+1$ non-isomorphic super edge-magic labelings.

Figure 18 depicts two different super edge-magic labelings of the disjoint union of five path-like trees obtained by applying the appropriate elementary transformations on $5 P_{6}$.

\section{Conclusions}

In this paper we introduced an algorithm that allows us to construct an exponential number of non-isomorphic strong super edge-magic labelings for $G \cong(2 K+1) P_{n}$. However at this point we do not know anything in general about the existence of strong super edge-magic labelings for the graph $G \cong(2 K) P_{n}$, except for the fact that $(2 K) P_{2}$ is not super edgemagic. To know an answer to this question is of great interest, since it would help us to understand the super edge-magic properties of an even union of path-like trees. Therefore we conclude this section with the following open problems. 
Open Problem 1 Let $G \cong(2 K) P_{n} ; n \neq 2$. Is $G$ a strong super edge magic?

Assuming that the answer to open problem 1 is yes, then it leads to open problem 2 .

Open Problem 2 Let $G \cong(2 K) P_{n} ; n \neq 2$. How many non-isomorphic strong super edge magic labelings does $G$ admit?

Open Problem 3 Let $G \cong \bigcup_{j=1}^{2 K} T_{j}$ be a union of an even number of path-like trees, all of them of the same order, and such that $T_{j} \neq P_{2}$ for $j=1,2, \ldots, 2 K$. Is $G$ a super edge-magic graph?

\section{References}

[1] J. Abrham and A. Kotzig, Exponential lower bounds for the number of graceful numbering of snakes, Congressus Numerantium, $\mathbf{7 2}$ (1990), 163-174.

[2] B.D. Acharya, Elementary parallel transformations of graphs, AKCE International J. of Graphs and Combinatorics 1 (2004), 63-67.

[3] R.E.L. Aldred, J. Širáň and M. Širáň, A note on the number of graceful labelings of paths, Discrete Math., 261 (2003), 27-30.

[4] M. Bača, Y. Lin and F. A. Muntaner-Batle, Normalized embeddings of path-like trees, Utilitas Math., To appear.

[5] M. Bača, Y. Lin and F. A. Muntaner-Batle, Edge-antimagic labelings of forest, Utilitas Math., To appear.

[6] M. Bača, Y. Lin and F. A. Muntaner-Batle, Super edge-antimagic labelings of the path-like trees, Utilitas Math. 73 (2007), 117-128.

[7] M. Bača and C. Barrientos, Graceful and edge-antimagic labelings, Ars Combin., to appear.

[8] C. Barrientos, Difference Vertex Labelings, Ph. D. Thesis. Universitat Politècnica de Catalunya (2004).

[9] M. Burzio and G. Ferrarese, The subdivision graph of a graceful tree is a graceful tree, Discrete Math., 181 (1998), 275-281. 
[10] H. Enomoto, A. Lladó, T. Nakamigawa and G. Ringel, Super edgemagic graphs, SUT J. Math. 34 (1998), 105-109.

[11] S.M. Hegde and S. Shetty, On graceful trees, Appl. Math. E-Notes 2 (2002), 192-197.

[12] G. Chartrand and L. Lesniak, Graphs and Digraphs, second edition. Wadsworth \& Brooks/Cole Advanced Books and Software, Monterey (1986).

[13] R.M. Figueroa-Centeno, R. Ichishima and F.A. Muntaner-Batle, The place of super edge-magic labelings among other classes of labelings, Discrete Math. 231(1-3) (2001), 153-168.

[14] R.M. Figueroa-Centeno, R. Ichishima, F.A. Muntaner-Batle and M. Rius-Font, Labeling generating matrices, J. Combin. Math. Combin. Comput., To appear.

[15] A. Kotzig and A. Rosa, Magic valuations of finite graphs, Canad. Math. Bull. 13 (1970), 451-461.

[16] F.A. Muntaner-Batle, Magic Graphs, Ph.D. Thesis, Departament de Matemática Aplicada IV, Universitat Politécnica de Catalunya, Barcelona, 2001.

[17] F. A. Muntaner-Batle and M. Rius-Font, On the structure of pathlike trees, Discussiones Math. Graph Theory, To appear.

[18] A.A.G. Ngurah, E.T. Baskoro and R. Simanjuntak, On edge-magic total labeling of $k C_{4}$-snakes and path-like trees, $M I H M I$, To appear.

[19] G. Ringel and A. Lladó, Another tree conjecture, Bull. Inst. Combin. Appl. 18 (1996), 83-85.

[20] A. Rosa, On certain valuations of the vertices of a graph, Theory of Graphs (Internat. Symposium, Rome, July 1966), Gordon and Breach, N.Y. and Dunod Paris (1967), 349-355.

[21] A. Rosa and J. Širán̆, Bipartite labelings of trees and the gracesize, J. Graph Theory, 19 (1995) 201-215.

[22] H. Snevily, New families of graphs that have $\alpha$-labelings, Discrete Math., 170 (1997), 185-194.

[23] W.D. Wallis, Magic Graphs, Birkhäuser, Boston (2001). 